\title{
Boolean-valued equivalence relations and complete extensions of complete boolean algebras
}

\section{Denis Higgs}

It is remarked that, if $A$ is a complete boolean algebra and $\delta$ is an $A$-valued equivalence relation on a non-empty set $I$, then the set of $\delta$-extensional functions from $I$ to $A$ can be regarded as a complete boolean algebra extension of $A$ and a characterization is given of the complete extensions which arise in this way.

Let $A$ be a boolean algebra, $I$ any non-empty set. An A-valued equivalence relation on $I$ is a function $\delta: I \times I \rightarrow A$ such that $\delta(i, i)=1, \delta(i, j)=\delta(j, i)$, and $\delta(i, j) \wedge \delta(j, k) \leq \delta(i, k)$ for all $i, j, k$ in $I$. Boolean-valued equivalence relations occur of course in boolean-valued model theory and in this context they were first introduced, so far as I know, by tos [5], p. 103. (As it happens, it is the complement $d(i, j)=\delta(i, j)^{\prime}$ of a boolean-valued equivalence relation which Łoś describes and he requires in addition that $d(i, j)=0$ only if $i=j$; such a function $d(i, j)$ may be regarded as an $A$-valued metric on $I$. Boolean-valued metrics have been considered by a number of authors - see, for example, Ellis and Sprinkle [1], p. 254.)

Given an $A$-valued equivalence relation $\delta$ on the non-empty set $I$, where from now on we suppose that the boolean algebra $A$ is complete, we

Received 28 ilarch 1970. Some of the results of this paper are drawn from the author's Ph.D. thesis "Matroids on complete boolean algebras" (McMaster University, 1970) written under the supervision of Dr G.O. Sabidussi. 
can consider the $\delta$-extensional functions from $I$ to $A$, that is, the functions $x$ in $A^{I}$ such that $x(i) \wedge \delta(i, j) \leq x(j)$ for all $i, j$ in $I$. It is easily seen that these functions form a complete subalgebra, which we denote by $B_{\delta}$, of the complete boolean algebra $A^{I}$;

furthermore $B_{\delta}$ contains the subalgebra $A_{1}$ of $A^{I}$ consisting of the constant functions from $I$ to $A$. Since $A_{1}$ is isomorphic to $A$, we can regard $B_{\delta}$ as a complete extension of $A$ and we wish to characterize the complete extensions which arise in this way.

The motivation for this is as follows. Even in the classical two-valued case we frequently find it convenient to present a set as the set $I / \delta$ of equivalence classes corresponding to an equivalence relation $\delta$ on some other set $I$ - in this case the algebra $B_{\delta}$ is seen to be isomorphic to the power set of $I / \delta$. In the general A-valued case we can still regard $B_{\delta}$ as giving the power set of the $A$-valued set $I / \delta$, where we now have to specify $B_{\delta}$, not just as a complete boolean algebra, but rather as a complete extension of the truth-value algebra $A$. The complete extensions thereby obtained provide an intrinsic, presentationfree description of such $A$-valued sets and it seems desirable to give an internal characterization of them.

We first introduce some notations and definitions. $A, B$, and $C$ will always denote complete boolean algebras. $A \leq B$ means that $A$ is a complete subalgebra of $B$. In the rest of this paragraph we shall suppose that $A$ and $B$ are given and satisfy $A \leq B$. Then $S(A, B)$ denotes the set of $C$ such that $A \leq C \leq B$ and $W(A, B)$ denotes the set of complete retractions from $B$ to $A$ (the ' $W$ ' here stands for 'witness' after Halmos [3], p. 244). We write $A \propto B$ and say that $A$ is an analytic subalgebra of $B$, or that $B$ is an analytic extension of $A$, iff $W(A, B)$ distinguishes the elements of $B$, equivalently, iff the evaluation mapping $e: B \rightarrow A^{W(A, B)}$, which in any case is a complete morphism, is one-to-one (cf. Grätzer [2], p. 155, Exercise 20). (We are going to show that the complete extensions of $A$ which arise as 
described above from $A$-valued equivalence relations are the same, to within isomorphism, as the analytic extensions of $A$.) Since $A \leq B$, we can define a quantifier $f=f_{A, B}$ on $B$ with $f(B)=A$ by putting $f(x)=\bigwedge\{a \in A ; a \geq x\}$. (A quantifier on a boolean algebra $B$ is a closure operator $f$ on $B$ such that $f(0)=0$ and $f(f(x) \wedge y)=f(x) \wedge f(y)$ for all $x, y$ in $B$ - see Halmos [3]; in the case, as here, that $B$ is complete, specifying a quantifier $f$ on $B$ is the same as specifying an $A \leq B$, the connection being the equation $f(B)=A$.) An element $s$ of $B$ is then said to be discrete if $f(x \wedge s) \wedge s \leq x \quad$ (equivalently, $f(x \wedge s) \wedge s=x \wedge s$ ) for all $x$ in $B$, and $s$ is said to be a base of an element $y$ of $B$ if $s$ is discrete and $s \leq y \leq f(s)$. $D(A, B)$ denotes the set of discrete elements and $B(A, B)$ denotes the set of bases of 1 . It is convenient to note here the following two simple facts. On account of $f(B)=A$ being a join-closed subset of $B, f$ is a join-preserving operator, that is, $f\left(V_{\lambda} x_{\lambda}\right)=V_{\lambda} f\left(x_{\lambda}\right)$ for any set $\{x ; \lambda \in \Lambda\}$ of elements of $B$ (Rubin [6], Theorem 1.3). Also if $w$ is in $W(A, B)$ then $w(x) \leq f(x)$ for all $x$ in $B$, as follows by applying $w$ to the inequality $x \leq f(x)$.

LEMMA 1. Let $A \leq B$ and let $\left\{s_{\lambda} ; \lambda \in \Lambda\right\}$ be a chain of elements of $O(A, B)$. Then $s=V_{\lambda} s_{\lambda}$ is in $D(A, B)$.

Proof. Let $x$ be any element of $B$. Then $f(x \wedge s) \wedge s=V_{\lambda, \mu}\left(f\left(x \wedge s_{\lambda}\right) \wedge s_{\mu}\right)=V_{\nu}\left(f\left(x \wedge s_{\nu}\right) \wedge s_{\nu}\right)$ since we have a chain, and this in turn is $\leq x$ since each $s_{\nu}$ is in $D(A, B)$. Thus $s$ is in $D(A, B)$.

LEMMA 2. Let $A \leq B$ and for each $s$ in $B(A, B)$ let $w_{s}: B \rightarrow A$ be defined by $w_{s}(x)=f(x \wedge s)$. Then the mappings $s \mapsto w_{s}$ and $w+\wedge w^{-1}(\{1\})$ set up a bijection between $B(A, B)$ and $w(A, B)$.

Proof. Let $s$ be in $B(A, B)$. Then $w_{s}(1)=f(s)=1$, $w_{s}\left(V_{\lambda} x_{\lambda}\right)=f\left(\left(V_{\lambda} x_{\lambda}\right) \wedge s\right)=V_{\lambda} f\left(x_{\lambda} \wedge s\right)=V_{\lambda} w_{s}\left(x_{\lambda}\right)$, and 
$w_{s}(x) \wedge w_{s}\left(x^{\prime}\right)=f(x \wedge s) \wedge f\left(x^{\prime} \wedge s\right)=f\left(f(x \wedge s) \wedge x^{\prime} \wedge s\right) \leq f\left(x \wedge x^{\prime}\right)$ $=0$.

It follows that $w_{s}$ is a complete morphism from $B$ to $A$. Also $w_{s}(f(x))=f(f(x) \wedge s)=f(x) \wedge f(s)=f(x)$ so that $w_{s}$ leaves $A$ elementwise fixed. Thus $w_{s}$ is in $W(A, B)$. Furthermore it is easy to see that $\wedge w_{s}^{-1}(\{1\})=\Lambda(x \in B ; f(x \wedge s)=1\}=s$.

Now take any $w$ in $W(A, B)$ and put $\wedge w^{-1}(\{1\})=s$. Then $w(s)=1$ and therefore $f(s)=1$. Let $x$ be an arbitrary element of $B$. Then $w(w(x) \Leftrightarrow x)=w(x) \Longleftrightarrow w(x)=1$ and hence $w(x) \Leftrightarrow x \geq s$, that is, $w(x) \wedge s=x \wedge s$. (N.B. For any elements $a, b$ of a boolean algebra $B, a \Longleftrightarrow b$ denotes the element $\left(a^{\prime} \vee b\right) \wedge\left(a \vee b^{\prime}\right)=(a \wedge b) \vee\left(a^{\prime} \wedge b^{\prime}\right)$ of $\left.B.\right)$ Therefore $f(x \wedge s)=f(w(x) \wedge s)=w(x) \wedge f(s)=w(x)$ (where the second equality holds because $w(x)$ is in $A)$ so that $f(x \wedge s) \wedge s=w(x) \wedge s=x \wedge s$. Thus $s$ is discrete and, since $f(s)=1, s$ is in $B(A, B)$. Moreover from the equality $f(x \wedge s)=w(x)$ we have $w_{s}=w$.

THEOREM 1. Let $A \leq B$. Then the following conditions are equivalent:

(i) $A \propto B$;

(ii) $V D(A, B)=1$;

(iii) every discrete subelement of an element $x$ of $B$ is contained in a base of $x$;

(iv) for each $x$ in $B$ there exists $w$ in $\omega(A, B)$ such that $w(x)=f(x)$ (where $\left.f=f_{A, B}\right)$.

REMARKS. ( $i i)$ is equivalent to the assertion that $D(A, B)$ is a join-dense subset of $B, D(A, B)$ being a lower section of $B$ (that is, $s \leq t$ and $t \in D(A, B)$ implies $s \in D(A, B))$.

(iii) is the condition used to define B-matroids in [4].

(iv) is the analogue for the complete monadic algebra $(B, f)$ of Halmos's notion of richness for monadic algebras in general ([3], p. 244). 
Proof. (i) implies ( $i i)$. If (i) holds then for each non-zero element $x$ of $B$ there exists, by Lemma 2 , an $s$ in $B(A, B)$ such that $f(x \wedge s) \neq 0$. But then $x \wedge s$ is a non-zero element of $D(A, B)$ contained in $x$. Hence $D(A, B)$ is join-dense in $B$.

(ii) implies (iii). Let $r$ be a discrete subelement of $x$. By Lemma 1 and Zorn's Lemma, $r$ is contained in some maximal discrete subelement $s$ of $x$. Given that (ii) holds, $s$ must be a base of $x$. For suppose $x \neq f(s)$ : then by (ii) there exists a non-zero discrete element $t \leq x \wedge f(s)^{\prime} ; f(s) \wedge t=0$ gives $f(s) \wedge f(t)=0$ from which it is not difficult to verify that $s \vee t$ is discrete, contrary to the maximality of $s$.

(iii) implies (iv). For $s$ in $B(A, B)$, the equation $w_{s}(x)=f(x)$ is equivalent to the assertion that $x \wedge s$ is a base of $x$ or, what is the same thing, that $s$ is an extension of a base of $x$ to a base of 1. The existence of such an $s$ for each $x$ in $B$ is an immediate consequence of $(i i)$ : extend 0 , which is certainly discrete, to a base of $x$ and then extend again to obtain a base of 1 .

It is trivial that (iv) implies (i).

COROLLARY. Let $A \leq B \leq C$. Then $A \propto C$ iff $A \propto B$ and $B \propto C$.

Proof. Since $u v$ is in $\omega(A, C)$ for $u$ in $\omega(A, B)$ and $v$ in $\omega(B, C)$, it is clear that $A \propto B$ and $B \propto C$ implies $A \propto C$; and $A \propto C$ implies $A \propto B$ since if $w$ is in $\omega(A, C)$ then $w \mid B$ is in $\omega(A, B)$. The remaining implication, from $A \propto C$ to $B \propto C$, is not so universal but follows from the equivalence of conditions $(i)$ and $(i i)$ in Theorem 1 , in view of the obvious inclusion $D(A, C) \subseteq D(B, C)$.

THEOREM 2. Let $A$ be a complete boolean algebra, I any non-empty set, and let $A_{1}$ be the set of constant functions in $A^{I}$.

(i) $A_{1} \propto B$ for each $B$ in $S\left(A_{1}, A^{I}\right)$.

(ii) For each $B$ in $S\left(A_{1}, A^{I}\right)$, define $\delta_{B}: I \times I \rightarrow A$ by $\delta_{B}(i, j)=\bigwedge_{x \in B} x(i) \leftrightharpoons x(j)$. Then the mappings $\delta \mapsto B_{\delta}$ and $B \mapsto \delta_{B}$ set up a bijection between the set of all A-valued equivalence relations $\delta$ 
on $I$ and $S\left(A_{1}, A^{I}\right)$.

Proof. In order to prove (i) it is sufficient, by the above corollary, to show that $A_{1} \propto A^{I}$. This latter statement follows from the

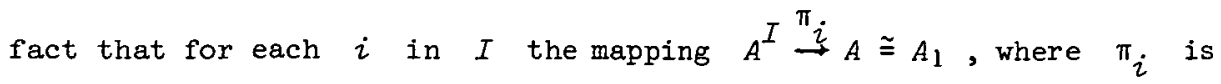
the $i$-th projection and $A \cong A_{l}$ is the obvious isomorphism, is in $\omega\left(A_{1}, A^{I}\right)$.

To obtain ( $i$ ), first take any $A$-valued equivalence relation $\delta$ on $I$ and put $B_{\delta}=B$. Then, as remarked earlier, $B$ is in $S\left(A_{1}, A^{I}\right)$. Furthermore $\delta_{B}=\delta$ since in the expression $\bigwedge_{x \in B} x(i) \Leftrightarrow x(j)$ for $\delta_{B}(i, j)$ we have $x(i) \Leftrightarrow x(j) \geq \delta(i, j)$ for all $x$ in $B$, with equality in the case $x(k) \equiv \delta(i, k)$.

Conversely, take any $B$ in $S\left(A_{1}, A^{I}\right)$ and put $\delta_{B}=\delta$. It is easy to see that $\delta$ is an $A$-valued equivalence relation on $I$ and that $B \subseteq B_{\delta}$. To obtain the reverse inclusion we prove

(A) If an element $s$ of $B_{\delta}$ satisfies $s(i) \wedge s(j) \leq \delta(i, j)$ for arz $i, j$ in $I$ then $s$ is in $D\left(A_{1}, B_{\delta}\right)$.

To see this, let $x$ be an arbitrary element of $B_{\delta}$. We require $f(x \wedge s) \wedge s \leq x$ and this is the case since for all $i$ in $I$ we have $(f(x \wedge s) \wedge s)(i)=\bigvee_{j} x(j) \wedge s(j) \wedge s(i) \leq \bigvee_{j} x(j) \wedge \delta(i, j) \leq x(i)$. (N.B. For any $C$ in $S\left(A_{1}, A^{I}\right)$, it is clear that the associated $f=f_{A_{1}, C}$ is given by $\left.(f(x))(i)=V_{j} x(j).\right)$

REMARK. It is not difficult to verify that the condition given in (A) is necessary, as well as sufficient, for $s$ to be in $D\left(A_{1}, B_{\delta}\right)$.

(B) $D\left(A_{1}, B\right) \subseteq D\left(A_{1}, B_{\delta}\right)$.

Let $s$ be in $D\left(A_{1}, B\right)$. Then, since $B \subseteq B_{\delta}, s$ is certainly in $B_{\delta}$. To show that $s(i) \wedge s(j) \leq \delta(i, j)$ for all $i, j$ in $I$ it is 
sufficient, in view of the way $\delta(i, j)$ was defined, to show that $s(i) \wedge s(j) \wedge x(i) \leq x(j)$ for all $x$ in $B$ and all $i, j$ in $I$ and this latter inequality follows easily from the fact that, since $s$ is in $D\left(A_{1}, B\right), f(x \wedge s) \wedge s \leq x$ for all $x$ in $B$.

We can now prove that $B_{\delta} \subseteq B$. Since $A_{1} \propto B$ by part (i) of the present result, we can write $I=V_{\lambda} s_{\lambda}$ where the $s_{\lambda}^{\prime}$ 's are in $D\left(A_{1}, B\right)$. Then if $x$ is in $B_{\delta}$ we have $x=V_{\lambda}\left(x \wedge s_{\lambda}\right)$. By (B), each $s_{\lambda}$ is in $D\left(A_{1}, B_{\delta}\right)$ and therefore $f\left(x \wedge s_{\lambda}\right) \wedge s_{\lambda}=x \wedge s_{\lambda}$. It follows that each $x \wedge s_{\lambda}$ is in $B$ and hence so is $x=V_{\lambda}\left(x \wedge s_{\lambda}\right)$. This completes the proof of Theorem 2 .

THEOREM 3. Let $A$ be a complete boolean algebra. Consider the complete extensions of $A$ obtained by taking an A-valued equivalence relation $\delta$ on a non-empty set $I$ and forming the algebra $B_{\delta}$ of $\delta$-extensional functions from $I$ to $A$ (by the identification of $A$ with the algebra $A_{1}$ of constant functions from $I$ to $A, B_{\delta}$ may be regarded as an extension of $A)$. Then to within isomorphisms leaving $A$ elementwise fixed, these complete extensions. of $A$ are precisely the analytic extensions of $A$.

Proof. By Theorem 2, part (i), $B_{\delta}$ is an analytic extension of $A_{1}$. On the other hand, if $B$ is any analytic extension of $A$ then the evaluation mapping $e: B \rightarrow A^{I}$, where $I=W(A, B)$, carries $A$ to $e(A)=A_{1}$ and $B$ to $e(B)$ in $S\left(A_{1}, A^{I}\right)$. By Theorem 2, part (ii), there is an $A$-valued equivalence relation $\delta$ on $I$ such that $B_{\delta}=e(B)$ and this gives the result.

\section{References}

[1] David Ellis and H.D. Sprinkle, "Topology of B-metric spaces", Compositio Math. $12(1954 / 56), 250-262$.

[2] George Grätzer, Universal algebra (Van Nostrand, Princeton, New Jersey, Toronto, London, Melbourne, 1968). 
[3] Paul R. Halmos, "Algebraic logic, I. Monadic boolean algebras", Compositio Math. $12(1954 / 56), 217-249$.

[4] D.A. Higgs, "Matroids and duality", Colloq. Math. 20 (1969), 215-220.

[5] Jerzy Łos, "Quelques remarques, théorèmes et problèmes sur les classes définissables d'algèbres". Mathematical interpretation of formal systems, 98-113. (North-Holland, Amsterdam, 1955).

[6] Jean E. Rubin, "Remarks about a closure algebra in which closed elements are open", Proc. Amer. Math. Soc. 7 (1956), 30-34.

University of Waterloo,

Water loo,

Ontario, Canada. 